\title{
Management of colon and rectal cancers during COVID-19 pandemic: A clinical guideline (TUMS-CRC-CoV19 Guideline)
}

\author{
Mohammad Reza Keramati ${ }^{1,2}$, Behnam Behboudi ${ }^{1,2}$, Seyed Mohsen Ahmadi-Tafti ${ }^{1,2}$, Alireza Kazemeini ${ }^{1,2}$, \\ Amir Keshvari ${ }^{1,2}$, Faeze Salahshour ${ }^{3}$, Mahdi Aghili ${ }^{4}$, Foroogh Alborzi ${ }^{5}$, Najmeh Aletaha ${ }^{5}$, Mohammad Babaei ${ }^{4}$, \\ Mohammad Naeem Bangash ${ }^{1}$, Nasser Ebrahimi-Daryani ${ }^{5}$, Amir Hossein Emami ${ }^{6}$, Farshid Farhan ${ }^{4}$, Peiman Haddad $^{4}$, \\ Mohammad Kalani ${ }^{5}$, Amirhosein Naseri ${ }^{1}$, Farhad Shahi ${ }^{6}$, Mohammad Sadegh Fazeli*1,2 $\mathbb{D}$
}

\section{Abstract}

Background: In a resource-demanding COVID-19 pandemic, guidelines can free up health care resources needed for providing better care to those with COVID-19 and other patients. This study was performed to design a guideline to manage patients with colorectal cancers during the COVID-19pandemic.

Methods: To design this guideline, major topics and headings of colon and rectal cancers (CRC) were selected and included. Based on the extent of COVID-19 infection in the community and availability of hospital resources, the guideline has been designed for 2 major COVID-19 phases. Several multidisciplinary discussion sessions were held to review the comments of experts, finalize the data, and write the guideline.

Results: This guideline has been prepared in 2 main COVID-19 phases of the community/hospital. Phase A refers to the condition where a large number of COVID-19 patients are admitted to the hospital, but limited surgical ICU beds and facilities are still accessible. In phase B, many people are affected by COVID-19, and all hospital resources are allocated for COVID 19 patients. In phase A, 4 major groups are discussed, including malignant and suspicious colorectal polyps, colon cancers, rectal cancers, and recurrent cancers. The approach to emergent cases, including obstruction, bleeding, and perforation, will be presented in phase B.

Conclusion: This guideline is a comprehensive instruction on the approach to colorectal cancers during the COVID-19 pandemic that covers the major topics of colon and rectal cancers in detail.

Keywords: Colon, Rectum, Neoplasm, COVID-19, Guideline

Conflicts of Interest: None declared

Funding: None

*This work has been published under CC BY-NC-SA 1.0 license.

Copyright $\odot$ Iran University of Medical Sciences

Cite this article as: Keramati MR, Behboudi B, Ahmadi-Tafti SM, Kazemeini A, Keshvari A, Salahshour F, Aghili M, Alborzi F, Aletaha N, Babaei M, Bangash MN, Ebrahimi-Daryani N, Emami AH, Farhan F, Haddad P, Kalani M, Naseri A, Shahi F, Fazeli MS. Management of colon and rectal cancers during COVID-19 pandemic: A clinical guideline (TUMS-CRC-CoV19 Guideline). Med J Islam Repub Iran. 2020 (29 Sep);34:128. https://doi.org/10.47176/mjiri.34.128

Corresponding author: Dr Mohammad Sadegh Fazeli, mhfazeli@sina.tums.ac.ir

1. Division of Colorectal Surgery, Department of Surgery, Tehran University of Medical Sciences, Tehran, Iran

2. Colorectal Surgery Research Center, Tehran University of Medical Sciences, Tehran, Iran

3. Department of Radiology, Tehran University of Medical Sciences, Tehran, Iran

4. Department of Radiation Oncology, Tehran University of Medical Sciences, Tehran, Iran

5. Division of Gastroenterology, Department of Internal Medicine, Tehran University of Medical Sciences, Tehran, Iran

6. Department of Hematology and Medical Oncology, Tehran University of Medical Sciences, Tehran, Iran $\uparrow$ What is "already known” in this topic:

In a resource-demanding COVID-19 pandemic, guidelines can free up health care resources needed for providing better care to COVID-19 and other patients. This study was performed to design a guideline to manage patients with colorectal cancers during the COVID-19 pandemic.

\section{$\rightarrow$ What this article adds:}

This guideline covers the management of colon and rectal cancers in different stages, malignant polyps, and recurrent colorectal cancers during the COVID-19 pandemic. It can help achieve better care for patients with colorectal cancers in the COVID-19 pandemic. Multidisciplinary team discussions play an important role in ensuring the best treatment for patients with colorectal cancer. 


\section{Introduction}

In January 2020, a novel coronavirus in the SARS-CoV phylogenetic clade was reported as the causative agent for the outbreak that occurred earlier in China in 2019. Later, the virus spread to many countries to become one of the most significant pandemics in the recent world history. The disease is now referred to as novel coronavirus disease 2019 or COVID-19 (1). During this pandemic, hospitals have encountered difficult decisions on preserving their clinical resources, including intensive care unit (ICU) beds and ventilators. To provide better care to COVID-19 patients and to manage available resources in the hospitals, they were advised to discontinue or postpone elective surgical operations (2).

Cancer patients are at a higher risk for infections, including COVID-19. Therefore, the risk of potential exposure to COVID-19 for these patients should be limited. Moreover, ensuring proper care for cancer patients, including those with colorectal cancers, is a major priority during the COVID-19 pandemic. Having an appropriate guideline for colorectal cancers, one of the most common human cancers, is essential in this setting.

This study was designed and performed at the division of colorectal surgery of Imam hospital, affiliated to Tehran University of Medical Sciences. This tertiary referral hospital is the largest teaching university hospital in the country with more than 1200 beds and a dedicated colorectal ward accepting colorectal patients nationwide. During COVID-19, more than 300 hospital beds, including colorectal beds, were filled with COVID-19 patients. The colorectal ward, including faculty members and staff, were involved with COVID-19 and colorectal cancer. A comprehensive and detailed guideline for better management of patients with colorectal cancer, including those who also had COVID-19, was mandatory in this setting. Reviewing the literature, we found a limited number of guidelines in this regard (2-4). Here, a comprehensive guideline based on a large-scale consensus and online virtual discussion panels is presented. This guideline is named as TUMS-CRC-CoV19 Guideline (Tehran University of Medical Sciences Colorectal Cancer COVID-19). In this guideline, the most common colorectal cancer topics are addressed.

\section{Methods}

This study was performed to design a guideline to manage patients with colorectal cancers during the COVID-19 pandemic. It was approved and conducted at the division of colorectal surgery of Imam hospital, which is affiliated to Tehran University of Medical Sciences (Tehran, Iran) from March 2020 to April 2020. Major topics and headings related to colon and rectal cancer were selected to design this guideline. Topics included colorectal cancers and malignant polyps. Subtopics in each subject were developed based on tumor staging and common referral cases.

Faculty members from top-ranking universities and university-affiliated hospitals were invited to take part in this survey. Invited faculty members included colorectal surgeons, gastroenterologists, oncologists, radiotherapists, and radiologists involved in colorectal cancer management. Experts were contacted by email. Data were collected using a questionnaire. The questions included topics of malignant polyps, colon cancers, rectal cancers, and recurrent colorectal cancers. Online virtual discussions were also done to cover all topics and subtopics. Virtual multidisciplinary discussion sessions were also held to review the comments and interpret the data.

Based on the extent of the COVID-19 infection in the community and the availability of hospital resources (including operating rooms and surgical ICUs), COVID-19 conditions were categorized into 2 phases (2). In phase A, many COVID-19 patients are admitted to the hospital. However, a limited number of surgical ICU beds and ventilators are still accessible. In community/hospital phase $\mathrm{B}$, many people in the community are affected with COVID-19, and all the hospital resources are directed toward the care of COVID 19 patients, with no surgical ICU beds and ventilators available at this time.

In addition to the designed guideline, a comprehensive review of available online guidelines and resources was done.

\section{Results}

This guideline has been prepared for 2 COVID-19 conditions according to the number of patients involved with COVID-19 in the community and hospital and the availability of hospital resources to care for patients following an operation, including operating rooms for colorectal surgery, beds, and ventilators in the surgical intensive care units (ICU).

\section{A - COVID-19 hospital/community phase A:}

Definition: Although many COVID-19 patients have been admitted to the hospital, surgical ICU beds and ventilators are available. The colorectal operating room is also ready. However, both the clinician and the patient should know that other patients with COVID-19 are admitted to the ICU and the ward at the same time.

Guideline: Recommendations in this setting have been categorized into 4 groups, including colorectal polyps, colon cancers, rectal cancers, and recurrent colorectal cancers. Approaches to emergent colorectal problems, such as obstruction, bleeding, and perforation will be mentioned in the COVID-19 phase B.

\section{A-1: Approach to malignant or suspicious colorectal polyps}

Below, we will present our recommendations on approaching malignant colorectal polyps case by case. Recommendations of this guideline and previous ones are summarized in Table 1.

A-1-1 - Colon or rectal polyps with large size or suspicious appearance found through colonoscopy

Recommendation: Treatment should be delayed until stable COVID-19 conditions (minimal or few numbers of COVID-19 patients in the hospital, complete availability of hospital resources, and low possibility of transmission of COVID-19 to the patient). 
Table 1. Malignant or suspicious colorectal polyps (Group A-1) - Our guideline and review of literature on currently available guidelines regarding the approach to malignant colorectal polyps in COVID-19 conditions.

\begin{tabular}{lcl}
\hline Sub-topic & Our Guideline Recommendations & \multicolumn{1}{c}{ Other Guidelines Recommendations } \\
\hline $\begin{array}{l}\text { Colon or rectal polyps with large } \\
\text { size or suspicious appearance on }\end{array}$ & Treatment should be deferred until & ACS: No recommendation \\
colonoscopy & stable COVID-19 conditions. * & NHS: Elective surgery can be delayed for 10-12 weeks and will \\
& & $\begin{array}{l}\text { have no predicted negative outcome. } \\
\text { ACPGBI: Defer the patient. Regular scheduled remote consulta- } \\
\text { tions followed by MDT case review to reassess prioritization. }\end{array}$
\end{tabular}

Colon polyp with $\mathrm{T}$ in-situ (or high-grade dysplasia) histology and involved margin

Rectal polyp with T in-situ (or high-grade dysplasia) pathology and involved margin

Malignant colon polyp without metastasis, with high-risk pathologic features

Malignant rectal polyp (T1N0M0) with high-risk pathologic features
Treatment should be deferred until stable COVID-19 conditions. *

Treatment should be deferred until stable COVID-19 conditions. *

Treatment should be deferred until stable COVID-19 conditions. * Possibility of neoadjuvant therapy should be discussed in MDT.

Treatment should be deferred until stable COVID-19 conditions. * Possibility of neoadjuvant therapy should be discussed in MDT.

ACS: No recommendation

NHS: Elective surgery can be delayed for 10-12 weeks and will have no predicted negative outcome.

ACPGBI: Defer the patient. Regular scheduled remote consultations followed by MDT case review to reassess prioritization.

ACS: No recommendation

NHS: Elective surgery can be delayed for 10-12 weeks and will have no predicted negative outcome.

ACPGBI: Defer the patient. Regular scheduled remote consultations followed by MDT case review to reassess prioritization.

ACS: Cases should be deferred OR Consider more local endoluminal therapies.

NHS: Elective surgery can be delayed for 10-12 weeks and will have no predicted negative outcome.

ACPGBI: Defer the patient. Regular scheduled remote consultations followed by MDT case review to reassess prioritization.

ACS: Cases should be deferred OR Consider more local endoluminal therapies.

NHS: Elective surgery can be delayed for 10-12 weeks and will have no predicted negative outcome.

ACPGBI: Defer the patient. Regular scheduled remote consultations followed by MDT case review to reassess prioritization.

ACS (2): American College of Surgeon

NHS (3): National Health Service of the United Kingdom

ACPGBI (4): Association of Coloproctology of Great Britain and Ireland

MDT: Multi-Disciplinary Team

* Stable COVID-19 condition is defined as minimal or few numbers of COVID-19 patients in the hospital, complete availability of hospital resources, and low transmission possibility of COVID-19 to the patient.

A-1-2 - Colon polyp with $\mathrm{T}$ in-situ (or high-grade dysplasia) histology and involved margin

Recommendation: Treatment should be delayed until stable COVID-19 conditions (minimal or few numbers of COVID-19 patients in the hospital, complete availability of hospital resources, and low possibility of transmission of COVID-19 to the patient). However, colonoscopic tattooing in case of availability can be done for an easier future follow-up.

A-1-3 - Rectal polyp with $\mathrm{T}$ in-situ (or high-grade dysplasia) pathology and involved margin

Recommendation: Treatment should be delayed until stable COVID-19 conditions (minimal or few numbers of COVID-19 patients in the hospital, complete availability of hospital resources, and low possibility of transmission of COVID-19 to the patient).

A-1-4 - Malignant colon polyp without metastasis, with high-risk histologic features $(>\mathrm{Sm} 2$, lymphovascular invasion, poorly differentiated, histologic grade $>=2$, involved margin $<1 \mathrm{~mm}$, sessile, incomplete or piecemeal resection).

Recommendation: In this condition, deferring treatment until stable COVID-19 condition is recommended. However, according to a multidisciplinary team, neoadjuvant therapy and delayed surgical operation are alternatives.

A-1-5 - Malignant rectal polyp (T1N0M0) with high- risk histologic features ( $>\mathrm{Sm} 2$, lymphovascular invasion, poorly differentiated, histologic grade $>=2$, involved margin $<1 \mathrm{~mm}$, sessile, incomplete or piecemeal resection).

Recommendation: Treatment should be delayed until stable COVID-19 conditions. Neoadjuvant chemoradiation, according to a multidisciplinary team discussion, can be offered in some cases.

\section{A-2: Approach to colon cancers}

Below, we will present our recommendations on approaching colon cancers. Recommendations of this guideline and previous ones are summarized in Table 2.

A-2-1 - Colon cancer without local invasion and metastasis

Recommendation: Treatment should be delayed until stable COVID-19 conditions.

A-2-2 - Colon cancer without local invasion and metastasis in patient with poor general health condition

Recommendation: These patients are at high risk for any kind of treatment, including surgery or chemotherapy. The risk of complications after interventions is very high. Chemotherapy can be discussed by a multidisciplinary team for these patients.

A-2-3 - Locally advanced colon cancer (T4) without metastasis

Recommendation: Depending on the patient's general 
Table 2. Colon cancer (Group A-2) - Our guideline and review of literature on currently available guidelines regarding the approach to colon cancer in COVID-19 conditions.

\begin{tabular}{lcl}
\hline Sub-topic & Our Guideline Recommendations & \multicolumn{1}{c}{ Other Guidelines Recommendations } \\
\hline $\begin{array}{l}\text { Colon cancer without local } \\
\text { invasion and metastasis }\end{array}$ & $\begin{array}{c}\text { Treatment should be deferred until stable } \\
\text { COVID-19 conditions. }{ }^{*}\end{array}$ & $\begin{array}{l}\text { ACS: Cases should be deferred OR Consider more local endo- } \\
\text { luminal therapies. }\end{array}$ \\
& $\begin{array}{l}\text { NHS: Elective curative surgery within } 4 \text { weeks based on the } \\
\text { symptoms. }\end{array}$ \\
& ACPGB: Defer the patient. Regular scheduled remote consul- \\
& tations followed by MDT case review to reassess prioritization.
\end{tabular}

\begin{abstract}
Colon cancer without local invasion and metastasis, in poor general health condition
\end{abstract}

Locally advanced colon cancer (T4) without metastasis

Colon cancer with resectable distant metastasis

\section{Chemotherapy should be discussed in MDT. ACS: No recommendation}

NHS: Treatment decisions will need to be made on a case-bycase basis with input from both patients and the MDT. ACPGBI: Supportive care if an expected life expectancy of less than 6 months.
Neoadjuvant chemotherapy (preferably oxaliplatin-based regimens) or neoadjuvant chemoradiation should be discussed in MDT.

Chemotherapy
ACS: Consider neoadjuvant therapy.

NHS: Treatment decisions will need to be made on a case-bycase basis with input from both patients and the MDT ACPGBI: NO recommendation

\section{ACS: No recommendation}

NHS: Treatment decisions to be made on a case-by-case basis with input from both patients and the MDT.

ACPGBI: A period of observation and self-isolation and reassessment when normal levels of service and access are available

Colon cancer with unresectable Chemotherapy should be discussed in MDT. distant metastasis

\section{ACS: No recommendation}

NHS: Treatment decisions will need to be made on a case-bycase basis with input from both patients and the MDT.

ACPGBI: Palliative chemotherapy if life expectancy is likely greater than 12 months.

\footnotetext{
ACS (2): American College of Surgeons

NHS (3): National Health Service of the United Kingdom

ACPGBI (4): Association of Coloproctology of Great Britain and Ireland

MDT: Multi-Disciplinary Team

* Stable COVID-19 condition is defined as minimal or few numbers of COVID-19 patients in the hospital, complete availability of hospital resources, and low transmission possibility of COVID-19 to the patient.
}

condition, the possibility of neoadjuvant chemotherapy (preferably oxaliplatin-based regimens) or neoadjuvant chemoradiation (based on the location of the tumor) should be discussed by a multidisciplinary team. Surgical resection is recommended later in a more stable COVID19 condition.

A-2-4 - Colon cancer with resectable distant metastasis

Recommendation: Chemotherapy is recommended for these patients. However, general health condition and other comorbidities of the patients should be considered.

A-2-5 - Colon cancer with unresectable distant metastasis

Recommendation: A multidisciplinary discussion is essential. Considering the risk-benefit ratio of chemotherapy, comorbidities and general health condition of the patient, chemotherapy can be considered.

\section{A-3: Approach to rectal cancers}

Below, we will present our recommendations on approaching rectal cancers. Recommendations of this guideline and previous ones are summarized in Table 3.

A-3-1 - Rectal cancer T1-2, N0, M0 without sphincter involvement

Recommendation: Deferring treatment until a more stable COVID-19 condition is the main recommendation for this group of patients. However, the possibility of neoadjuvant chemoradiation (short or long course) should be discussed by a multidisciplinary team, and the operation can be postponed for some months. A complete clinical response might occur after the chemoradiation, which can be helpful.

A-3-2 - Rectal cancer T1-2, N0, M0 with sphincter involvement

Recommendation: Neoadjuvant chemoradiation is recommended for these patients. After neoadjuvant therapy, surgical resection can be done later until stable COVID-19 conditions. However, deferring the whole treatment to more stable conditions is another option. A complete clinical response might occur after the chemoradiation, which can be helpful.

A-3-3 - Rectal cancer T3, N0, M0 without sphincter involvement

Recommendation: Neoadjuvant chemoradiation is recommended for these patients. A decision on short or long course chemoradiation should be made based on your plan for future surgical operation and considering the status of COVID-19 in your hospital and community. It should be discussed by a multidisciplinary team.

A-3-4 - Rectal cancer T3, N0, M0 with sphincter involvement

Recommendation: Neoadjuvant chemoradiation is recommended for these patients. A decision on short or long course chemoradiation should be made based on your plan for future surgical operation and considering the status of COVID-19 in your hospital and community. It should be discussed by a multidisciplinary team. 
Table 3. Rectal cancer (Group A-3) - Our guideline and review of literature on currently available guidelines regarding the approach to rectal cancer in COVID-19 conditions.

\begin{tabular}{lc}
\hline Sub-topic & $\begin{array}{c}\text { Our Guideline Recommenda- } \\
\text { tions }\end{array}$ \\
\hline $\begin{array}{l}\text { Rectal cancer T1-2, N0, M0 } \\
\text { without sphincter involve- } \\
\text { ment }\end{array}$ & $\begin{array}{c}\text { Treatment should be deferred } \\
\text { until stable COVID-19 condi- } \\
\text { tions. * }\end{array}$ \\
& $\begin{array}{c}\text { Possibility of neoadjuvant } \\
\text { chemoradiation should be dis- } \\
\text { cussed in MDT. }\end{array}$ \\
$\begin{array}{l}\text { Rectal cancer T1-2, N0, M0 } \\
\text { with sphincter involvement }\end{array}$ & Neoadjuvant chemoradiation
\end{tabular}

Rectal cancer T3, N0, M0

Neoadjuvant chemoradiation

ACS: Cases should be deferred OR Consider neoadjuvant therapy.

Other Guidelines Recommendations

without sphincter involve-

ment

NHS: Elective curative surgery within 4 weeks based on the symptoms.

ACPGBI: Treatment may be delayed 3 to 4 months if necessary.

ACS: Cases should be deferred OR Consider neoadjuvant therapy.

NHS: Elective curative surgery within 4 weeks based on the symptoms. ACPGBI: Chemoradiotherapy.

ACS: Consider neoadjuvant therapy.

NHS: Treatment decisions will need to be made on a case-by-case basis with input from both patients and the MDT.

ACPGBI: If resources allow, short-course radiotherapy (SCRT) as an initial definitive treatment OR treatment may be deferred

Rectal cancer T3, N0, M0 with sphincter involvement

Neoadjuvant chemoradiation

ACS: Consider neoadjuvant therapy.

NHS: Treatment decisions will need to be made on a case-by-case basis with input from both patients and the MDT.

ACPGBI: Chemoradiotherapy.

Rectal cancer $\mathrm{T} 1-3, \mathrm{~N}+, \mathrm{M} 0$ without sphincter involvement

Neoadjuvant chemoradiation

ACS: Consider neoadjuvant therapy.

NHS: Treatment decisions will need to be made on a case-by-case basis with input from both patients and the MDT.

ACPGBI: Neoadjuvant radiotherapy (short-course radiotherapy, SCRT )

Rectal cancer $\mathrm{T} 1-3, \mathrm{~N}+, \mathrm{M} 0$

Neoadjuvant chemoradiation with sphincter involvement

Surgically resectable locally advanced rectal cancer (T4, $\mathrm{M} 0)$

Neoadjuvant chemoradiation

ACS: Consider neoadjuvant therapy.

NHS: Treatment decisions will need to be made on a case-by-case basis with input from both patients and the MDT.

ACPGBI: Chemoradiotherapy

ACS: Consider neoadjuvant therapy.

NHS: Treatment decisions will need to be made on a case-by-case basis with input from both patients and the MDT.

ACPGBI: Chemoradiotherapy.

Surgically unresectable locally advanced rectal cancer (T4, M0)

Neoadjuvant chemoradiation

ACS: NO recommendation

NHS: Treatment decisions will need to be made on a case-by-case basis with input from both patients and the MDT.

ACPGBI: Early supportive care, Enforced observation, Refer the patient, Discussion in a specialist MDT

Rectal cancer with resectable distant metastasis $(\mathrm{M}+)$

Chemotherapy

Rectal cancer with unresectable distant metastasis( $\mathrm{M}+)$
Chemotherapy should be discussed in MDT.
NHS: Treatment decisions will need to be made on a case-by-case basis
ACS: NO recommendation with input from both patients and the MDT.

ACPGBI: A period of observation and self-isolation and reassessment when normal levels of service and access are available

ACS: NO recommendation

NHS: Treatment decisions will need to be made on a case-by-case basis with input from both patients and the MDT.

ACPGBI: Palliative chemotherapy if life expectancy is likely greater than 12 months

ACS (2): American College of Surgeons

NHS (3): National Health Service of the United Kingdom

ACPGBI (4): Association of Coloproctology of Great Britain and Ireland

MDT: Multi-Disciplinary Team

* Stable COVID-19 condition is defined as minimal or few numbers of COVID-19 patients in the hospital, complete availability of hospital resources, and low transmis-

sion possibility of COVID-19 to the patient.

A-3-5 - Rectal cancer T1-3, N+, M0 without sphincter involvement

Recommendation: Neoadjuvant chemoradiation is recommended for these patients. A decision on short or long course chemoradiation should be made based on your plan for future surgical operation and considering the status of COVID-19 in your hospital and community. It should be discussed by a multidisciplinary team.
A-3-6 - Rectal cancer T1-3, N+, M0 with sphincter involvement

Recommendation: Neoadjuvant chemoradiation is recommended for these patients. A decision on short or long course chemoradiation should be made based on your plan for future surgical operation and considering the status of COVID-19 in your hospital and community. It should be discussed by a multidisciplinary team. 
A-3-7 - Surgically resectable locally advanced rectal cancer (T4, M0)

Recommendation: Neoadjuvant chemoradiation is recommended for these patients. A decision on short or long course chemoradiation should be made based on your plan for future surgical operation and considering the status of COVID-19 in your hospital and community. It should be discussed by a multidisciplinary team.

A-3-8 - Surgically unresectable locally advanced rectal cancer (T4, M0)

Recommendation: Neoadjuvant chemoradiation is recommended for these patients. A decision on short or long course chemoradiation should be made based on your plan for future surgical operation and considering the status of COVID-19 in your hospital and community. It should be discussed by a multidisciplinary team.

A-3-9 - Rectal cancer with resectable distant metasta$\operatorname{sis}(\mathrm{M}+)$

Recommendation: Chemotherapy is the preferred option for these patients.

A-3-10 - Rectal cancer with unresectable distant metas$\operatorname{tasis}(\mathrm{M}+)$

Recommendation: Chemotherapy is the preferred option for these patients. However, the risk-benefit ratio of chemotherapy with respect to the general health condition of the patient in the COVID-19 situation should be assessed by a multidisciplinary team because the treatment can be delayed until a more stable COVID-19 condition presents in your hospital and community.

\section{A-4: Approach to recurrent colorectal cancers}

Below, we will present our recommendations on approaching recurrent colorectal cancers. Recommendations of this guideline and previous ones are summarized in Table 4.

A-4-1 - Resectable local recurrence of a previously treated colon cancer

Recommendation: Chemotherapy is recommended for these patients.

A-4-2 - Resectable distant recurrence (metastasis) from a previously treated colon cancer

Recommendation: Chemotherapy is recommended for these patients.

A-4-3 - Unresectable local/distant recurrence from a previously treated colon cancer

Recommendation: Possibility and safety of chemotherapy should be discussed for these patients in a multidisciplinary team. According to the location of the tumor, radiotherapy can be an alternative for local recurrences. In the discussion panel, the general health condition of the patient, survival of the patient, and COVID-19 status should be considered as palliative care is another option.

A-4-4 - Resectable local pelvic recurrence of a previously treated rectal cancer

Recommendation: Considering the previous history of radiotherapy in the patient, chemotherapy, or chemoradiation are the 2 available alternatives. A final decision should be based on a multidisciplinary discussion panel, considering the COVID-19 condition in your hospital and

Table 4. Recurrent colorectal cancers (Group A-4) - Our guideline and review of literature on currently available guidelines regarding the approach to recurrent colorectal cancers in COVID-19 conditions.

\begin{tabular}{|c|c|c|}
\hline Sub-topic & Our Guideline Recommendations & Other Guidelines Recommendations \\
\hline $\begin{array}{l}\text { Resectable local recurrence of a } \\
\text { previously treated colon cancer }\end{array}$ & Chemotherapy & $\begin{array}{l}\text { ACS: NO recommendation } \\
\text { NHS: Treatment decisions will need to be made on a case-by-case } \\
\text { basis with input from both patients and the MDT. } \\
\text { ACPGBI: NO recommendation. }\end{array}$ \\
\hline $\begin{array}{l}\text { Resectable distant recurrence } \\
\text { (metastasis) from a previously } \\
\text { treated colon cancer }\end{array}$ & Chemotherapy & $\begin{array}{l}\text { ACS: NO recommendation } \\
\text { NHS: Treatment decisions will need to be made on a case-by-case } \\
\text { basis with input from both patients and the MDT. } \\
\text { ACPGBI: A period of observation and self-isolation and reassessment } \\
\text { when normal levels of service and access are available. }\end{array}$ \\
\hline $\begin{array}{l}\text { Unresectable local/distant recur- } \\
\text { rence from a previously treated } \\
\text { colon cancer }\end{array}$ & $\begin{array}{l}\text { Chemotherapy should be dis- } \\
\text { cussed in MDT. }\end{array}$ & $\begin{array}{l}\text { ACS: NO recommendation } \\
\text { NHS: Treatment decisions will need to be made on a case-by-case } \\
\text { basis with input from both patients and the MDT. } \\
\text { ACPGBI: Palliative chemotherapy if life expectancy is likely greater } \\
\text { than } 12 \text { months }\end{array}$ \\
\hline $\begin{array}{l}\text { Resectable local pelvic recurrence } \\
\text { of a previously treated rectal cancer }\end{array}$ & $\begin{array}{l}\text { Chemotherapy or chemoradiation } \\
\text { based on previous history of } \\
\text { radiotherapy in the patient. }\end{array}$ & $\begin{array}{l}\text { ACS: NO recommendation } \\
\text { NHS: Treatment decisions will need to be made on a case-by-case } \\
\text { basis with input from both patients and the MDT. } \\
\text { ACPGBI: Early supportive care, Enforced observation, Refer the } \\
\text { patient, Discussion in a specialist MDT. }\end{array}$ \\
\hline $\begin{array}{l}\text { Resectable distant recurrence } \\
\text { (metastasis) from a previously } \\
\text { treated rectal cancer }\end{array}$ & Chemotherapy & $\begin{array}{l}\text { ACS: NO recommendation } \\
\text { NHS: Treatment decisions will need to be made on a case-by-case } \\
\text { basis with input from both patients and the MDT. } \\
\text { ACPGBI: A period of observation and self-isolation and reassessment } \\
\text { when normal levels of service and access are available }\end{array}$ \\
\hline $\begin{array}{l}\text { Unresectable local/distant recur- } \\
\text { rence from a previously treated } \\
\text { rectal cancer }\end{array}$ & $\begin{array}{l}\text { Chemotherapy should be } \\
\text { discussed in MDT. }\end{array}$ & $\begin{array}{l}\text { ACS: NO recommendation } \\
\text { NHS: Treatment decisions will need to be made on a case-by-case } \\
\text { basis with input from both patients and the MDT } \\
\text { ACPGBI: Palliative chemotherapy if life expectancy is likely greater } \\
\text { than } 12 \text { months }\end{array}$ \\
\hline
\end{tabular}

ACS (2): American College of Surgeons

NHS (3): National Health Service of the United Kingdom

ACPGBI (4): Association of Coloproctology of Great Britain and Ireland

MDT: Multi-Disciplinary Team 
community.

A-4-5 - Resectable distant recurrence (metastasis) from a previously treated rectal cancer

Recommendation: Chemotherapy is recommended for these patients.

A-4-6 - Unresectable local/distant recurrence from a previously treated rectal cancer

Recommendation: Possibility and safety of chemotherapy should be discussed for these patients by a multidisciplinary team. General health condition of the patient, survival of the patient, and COVID-19 status should be considered as palliative care is another option.

\section{B - COVID-19 hospital/community phase B:}

Definition: Many people in the community and your hospital are infected by COVID-19, and all hospital resources are directed toward the care of COVID-19 patients; no ICU bed and ventilator are available at this time.

Guideline: Patients with colorectal cancer in which death is likely within hours should only be considered in this condition, including obstruction, bleeding, and perforation. All other problems should differ until a more stable situation in your hospital and/or community. Other patients can also be transferred or referred to nearby hospitals with better COVID-19 conditions if available. The following cases need emergent management in this phase.

\section{B-1: Colorectal cancer presented with complete ob-} struction

Recommendation: In the case of complete colorectal obstruction, the surgical operation should be considered as soon as possible. Obstructing colon or rectal cancers should be diverted using a stoma. Resection is not recommended as it exposes the patient to longer operation time and potential more postoperative risks. Partially obstructive colon and rectal cancers can be managed nonsurgically just for a limited time of fewer than 24 hours. Afterward, they need an urgent diversion. For partial rectal obstructing tumors, stenting is an option, if available.

\section{B-2: Colorectal cancer presented with bleeding}

Recommendation: If the patient is stable and is not transfusion-dependent, bleeding is recommended to be controlled using nonsurgical measures, proper hydration, and blood transfusion. Local endoluminal therapies (such as argon plasma coagulation or epinephrine injection) to control the bleeding may be helpful in these patients. However, an emergent surgical operation should be warranted for unstable patients requiring a massive blood transfusion. Operation in these patients includes resection of the bleeding segment and proximal stoma (Hartman's procedure). An anastomosis is not recommended in these unstable patients with a history of the previous transfusion.

\section{B-3: Colorectal cancer presented with perforation}

Recommendation: Emergent surgical operation is recommended for these patients after proper hydration. Based on the location of the primary tumor (colon or rectum) and site of the perforation (within the tumor or proximal to the tumor), the surgical approach is different. Irrigation of the peritoneal cavity and limited segmental resection of the perforated segment and proximal stoma are the recommended operations. However, if the tumor is perforated, tumor resection should be considered as well. Distal mucous fistula is also proposed to vent the distal segment if needed. An anastomosis is not recommended in these patients, even in stable patients with negligible intraabdominal contamination.

\section{Discussion}

Clinical guidelines are essential parts of clinical practice to improve the quality of clinical decisions, the quality of care, and health outcomes received by patients. According to the Institute of Medicine, clinical guidelines are defined as "systematically developed statements to assist practitioner and patient decisions about appropriate health care for specific clinical circumstances." (5) In a resourcelimited health care system, such as COVID-19 conditions, guidelines can free up resources needed for providing better care to COVID-19 patients. In this special setting, guidelines can offer recommendations on how to proceed with other routine and elective patients and improve the consistency of care for them (6).

In a condition like the COVID-19 pandemic, special decisions should be made to manage cancer patients. Although cancer surgery is essential in general, it may need to be delayed as hospitals are forced to preserve necessary resources for the care of critically ill patients during the pandemic. It has been mentioned that cancer patients have a significantly higher risk of severe infection with COVID-19 if they get admitted. This risk increases after chemotherapy or surgery. Conversely, the potential harms of deferring cancer treatment should be discussed by the clinician and the patient. In general, patients should receive proper surgical care and operative management, based on sound clinical judgment and availability of resources. Deciding on whether to perform elective surgical operations, we must consider the available resources of our local health center. Nonoperative management should also be considered if clinically appropriate $(2,7)$. Choosing the best surgical approach for cancer patients in COVID-19 conditions, one should choose a strategy to minimize the operating time and maximize the safety of patients and health care staff. There are insufficient data to compare open and laparoscopic surgical procedures for cancer patients (8). Virtual technologies, including tumor boards, and multidisciplinary discussion panels, are helpful in making an appropriate decision for patients with cancer. According to our guideline, most of the critical decisions are preferably taken according to the multidisciplinary team discussion.

Colonoscopic interventions can be helpful in managing patients with colorectal cancer. A primary concern regarding colonoscopy in COVID-19 patients is the risk of device and personnel infection from intestinal secretions since the active virus might be transmitted through intestinal discharges (9). Moreover, the smoke and gas emitted during colonoscopy can spread viral particles and therefore become a hazard (10). It has also been recommended 
that health care workers performing aerosol-generating procedures should use fitted respirator masks (11). Routine cancer screenings should also be postponed to prevent patients from contact with possible infected facilities. Therefore, we recommend withholding all elective colonoscopies in the current situation; however, based on a multidisciplinary team discussion, it can be offered in some cases if necessary.

According to our guideline, radiation therapy, including chemoradiation, is a good alternative for some rectal cancers when facing limited hospital resources in COVID-19 conditions. Also, screening the patients for signs and symptoms of COVID-19 or recent contact with infected persons has been recommended (12). In case of suspicion, a chest computer tomography (CT) scan and laboratory exams have been shown to be more sensitive than RTPCR (13). In case of any evidence of coronavirus infection or suspicious symptoms, it is advised to terminate the treatment (14). In the COVID-19 condition, short-course radiation therapy (SCRT) is believed to be as effective as conventional radiotherapy (15). SCRT reduces the number of radiation sessions, which is much safer for the patients and staff. A complete clinical response following radiation therapy, including SCRT, is also an excellent chance for the elderly who are at considerable risk of infections, such as COVID-19, compared to surgery complications (16).

Chemotherapy both increases the risk of infection with COVID-19 and is a threat for severe disease (7). The nature of infection might differ in immune-compromised patients. In addition to fever and shortness of breath like other COVID-19 patients, occasional coughs might be the only sign that can rapidly exacerbate the patients' condition (17). However, according to our guideline, chemotherapy can be a good alternative for patients with colorectal cancer who are at a high-risk for infectious postoperative complications during the COVID-19 pandemic.

Preoperative and intraoperative cautions and protective measures for both the patients and health care personnel should be considered as well. Patients with signs of infection should have a laboratory examination before the operation, including complete blood count, C-reactive protein, erythrocyte sedimentation rate, lactate dehydrogenase, and creatinine $(18,19)$. We recommend a chest CT scan before any major colorectal operation; if suspected, a real time polymerase chain reaction (RT-PCR) test is suggested. Intubated patients who are suspected of COVID19 infection should also have endotracheal aspirates sampled (11). In the operating room, closed system suctions are preferred over central suctions (20). Personnel should wear coverall gowns to protect themselves from COVID19 positive patients; also, it is better to use double gloves when in contact with bodily fluids during the operation (21).

\section{Conclusion}

During the COVID-19 pandemic, proper management of colorectal cancer, one of the most common cancers in the world, is difficult. As doctors, we have a responsibility to ensure essential service care to both cancer and noncancer patients in situations in which all hospital resources are routed to COVID-19 patients. Here, we have designed and presented a comprehensive guideline for approaching colorectal cancers in COVID-19 conditions. The guideline covers the management of colon and rectal cancers in different stages, malignant polyps, and recurrent colorectal cancers. We have also tried to recommend the best treatment options for each condition, including surgery, radiation therapy, chemotherapy, or observations based on their respective risks and benefits. Multidisciplinary team discussion plays an important role in this guideline so as to ensure the best treatment for patients with colorectal cancer.

\section{Conflict of Interests}

The authors declare that they have no competing interests.

\section{References}

1.European Centre for Disease Prevention and Control. Infection prevention and control for COVID-19 in healthcare settings [ECDC web site]. 2020, March 12 [Available from: https://www.ecdc.europa.eu/en/publications-data/infection-preventionand-control-covid-19-healthcare-settings.

2. American College of Surgeons. COVID-19 Guidelines for Triage of Colorectal Cancer Patients [ACS web site]. 2020, March 24 [Available from: https://www.facs.org/covid-19/clinical-guidance/elective-case/ colorectal-cancer.

3. National Health Service. Clinical guide for the management of surgical patients during the coronavirus pandemic [NHS web site]. 2020, March 16 [Available from: https://www.england.nhs.uk/coronavirus/ publication/specialty-guides/.

4. Association of Coloproctology of Great Britain and Ireland. Considerations for Multidisciplinary Management of Patients with Colorectal Cancer during the COVID- 19 Pandemic [ACPGBI web site] 2020, March 26 [Available from: https://www.acpgbi.org.uk/news/considerations-for-multidisciplinarymanagement-of-patients-with-colorectal-cancer-during-the-covid-19pandemic/.

5. Field MJ, Lohr KN. Definitions of Key Terms. In: Field MJ, Lohr KN, editors. Clinical Practice Guidelines: Directions for a New Program. Washington (DC) 1990 .

6. Woolf SH, Grol R, Hutchinson A, Eccles M, Grimshaw J. Clinical guidelines: potential benefits, limitations, and harms of clinical guidelines. BMJ. 1999;318(7182):527-30.

7.Liang W, Guan W, Chen R, Wang W, Li J, Xu K, et al. Cancer patients in SARS-CoV-2 infection: a nationwide analysis in China Lancet Oncol. 2020;21(3):335-7.

8. Society of American Gastrointestinal and Endoscopic Surgeons SAGES and EAES Recommendations Regarding Surgical Response to COVID-19 Crisis [SAGES web site] 2020, March 29 [Available from: https://www.sages.org/recommendations-surgical-response-covid-19/.

9. Leung WK, To KF, Chan PK, Chan HL, Wu AK, Lee N, et al. Enteric involvement of severe acute respiratory syndrome-associated coronavirus infection. Gastroenterology. 2003;125(4):1011-7.

10. Yu GY, Lou Z, Zhang W. [Several suggestion of operation for colorectal cancer under the outbreak of Corona Virus Disease 19 in China]. Zhonghua Wei Chang Wai Ke Za Zhi. 2020;23(3):9-11.

11. Alhazzani W, Moller MH, Arabi YM, Loeb M, Gong MN, Fan E, et al. Surviving Sepsis Campaign: guidelines on the management of critically ill adults with Coronavirus Disease 2019 (COVID-19). Intensive Care Med. 2020;46(5):854-87

12. Rivera A, Ohri N, Thomas E, Miller R, Knoll MA. The Impact of COVID-19 on Radiation Oncology Clinics and Cancer Patients in the U.S. Adv Radiat Oncol. Epub ahead of print 27 March 2020. DOI: 10.1016/j.adro.2020.03.006.

13. Xie C WX, Liu H, Bao Z, Yu J, Zhong Y, Chua MLK. Infection Control of 2019 Novel Corona Virus Disease (COVID-19) in Cancer Patients undergoing Radiotherapy in Wuhan [Medrxiv web site]. 2020, March 24 [Available from: https://www.medrxiv.org/content/10.1101/ 2020.03.21.20037051v1.full.pdf. 
14. Krengli M, Ferrara E, Mastroleo F, Brambilla M, Ricardi U. Running a Radiation Oncology Department at the time of coronavirus: an Italian experience. Adv Radiat Oncol. Epub ahead of print 20 March 2020. DOI: $10.1016 /$ j.adro.2020.03.003.

15. Ngan SY, Burmeister B, Fisher RJ, Solomon M, Goldstein D, Joseph

$\mathrm{D}$, et al. Randomized trial of short-course radiotherapy versus longcourse chemoradiation comparing rates of local recurrence in patients with T3 rectal cancer: Trans-Tasman Radiation Oncology Group trial 01.04. J Clin Oncol. 2012;30(31):3827-33.

16. Cummings MA, Usuki KY, Fleming FJ, Tejani MA, Katz AW. Short course radiation therapy for rectal cancer in the elderly: can radical surgery be avoided? J Gastrointes Oncol. 2019;10(2):357.

17. Ju CR, Lian QY, Zhang JH, Qiu T, Cai ZT, Jiang WY, et al. Recommended prophylactic and management strategies for severe acute respiratory syndrome coronavirus 2 infection in transplant recipients. Chronic Dis Transl Med. Epub ahead of print 27 March 2020. DOI: $10.1016 /$ j.cdtm.2020.02.003.

18. Zhang HY, Wang LW, Chen YY, Shen XK, Wang Q, Yan YQ, et al. A Multicentre Study of 2019 Novel Coronavirus Disease Outcomes of Cancer Patients in Wuhan, China [Medrxiv web site]. 2020, April 15 [Available from: https://www.medrxiv.org/content/10.1101/ 2020.03.21.20037127v2.full.pdf

19. Sohrabi C, Alsafi Z, O'Neill N, Khan M, Kerwan A, Al-Jabir A, et al. World Health Organization declares global emergency: A review of the 2019 novel coronavirus (COVID-19). Int J Surg. 2020;76:71-6.

20. Huang J, Hu Y, Wang J, Wang $\mathrm{D}$, Zhu Z, Hu Z, et al. Recommendation about the perioperative prevention of infection to healthcare workers and the anesthesia management of children with SARS-CoV-2 infection. World J Pediatr Surg. 2020;3(1):e000126.

21. Chen X, Shang Y, Yao S, Liu R, Liu H. Perioperative care provider's considerations in managing patients with the COVID-19 infections. Transl Perioper Pain Med. 2020;7:216-24. 